\title{
Thin plate spline interpolation on an annulus
}

\author{
David R. Jenkins*
}

(Received 7 August 2000)

\begin{abstract}
This work demonstrates a method for surface interpolation on an annulus, with the additional constraint that the surface and its normal derivatives are specified on the annulus boundaries. The method is based on a truncated Fourier series solution to the homogeneous biharmonic equation, combined with Green's functions for the point interpolations. The formulation and solution approach is particularly suitable for the annulus geometry because the boundaries are circles, meaning that the boundary constraints are functions of the angle $\theta$
\end{abstract}

* CSIRO Mathematical and Information Sciences, Locked Bag 17, North Ryde, NSW 1670 Australia.

${ }^{0}$ See http://anziamj . austms . org.au/V42/CTAC99/Jenk for this article and ancillary services, (c) Austral. Mathematical Soc. 2000. Published 27 Nov 2000. 
only, and they can be approximated by a truncated Fourier series. The result is that the interpolation method is effectively gridless. The matrix problem resulting from this formulation is highly structured and can be solved in a simple sequential manner. We present results for some test problems to illustrate the effect of the truncation of the Fourier series on the solution and on aspects such as the condition of the matrix problem.

\section{Contents}

1 Introduction

C821

2 Development of solution

C822

3 Interpolation on a circle

C828

4 Examples

C829

5 Conclusion

C835

References

C836 


\section{Introduction}

Suppose that one wishes to find a function that interpolates a set number of points in a finite region of two-dimensional space, and also satisfies conditions on the function and its normal derivative at the boundaries of the region. A desirable feature of such an interpolation function is that it be smooth, in some sense. Suppose, further, that the region of interest is an annulus. Such a situation arose in a recent industrial contact.

A common approach to smooth interpolation in two-dimensions is the so-called "thin-plate spline". A good description of thin-plate spline interpolation is given by Green \& Silverman [2]. They discuss the application of thin-plate splines in the case where boundary domains are ignored. In many cases, the representation of the interpolating function is only of interest within the convex hull of the interpolation points. In the thin-plate analogy, this is equivalent to a plate which extends to infinity. Dyn \& Levin [1] and Stone [4] have considered the effect of finite domain boundaries, by applying "natural" boundary conditions that arise from the thin-plate spline formulation. In the plate analogy, this is equivalent to a finite plate that is "free" or unconstrained along its edges. In our case, where we want to define conditions on the function and its normal derivative at the boundaries, the appropriate analogy is of a finite plate which is "clamped" around its edges. We have been unable to find any published information on such a problem.

The annular geometry is interesting in its own right, because it has an 
internal boundary as well as an external one. The work of Stone [4] and most other papers on surface interpolation (see Hegland et al [3], Wessel \& Bercovici [5] for recent examples) only consider simple polygonal domains, but the internal boundary allows for a wider set of basis functions for the thinplate spline. The other useful feature about an annulus is that its boundaries are circles, and its geometry lends itself to simple representations of solutions to the biharmonic equation, which is fundamental to the development of thinplate spline solutions.

\section{Development of solution}

We want to develop a smooth surface

$$
z=f(\mathbf{x})
$$

in $\mathbf{R}^{2}$, defined on the annulus $\boldsymbol{\Omega}$,

$$
\boldsymbol{\Omega}=\left\{\mathbf{x} \mid \mathbf{x}=(r, \theta), r_{1} \leq r \leq r_{2}\right\}
$$

that interpolates a set of $M$ points

$$
\left\{\mathbf{x}_{m}=\left(r_{m}, \theta_{m}\right), m=1, \ldots, M \mid r_{1}<r_{m}<r_{2}, f\left(\mathbf{x}_{m}\right)=z_{m}\right\}
$$

in a smooth fashion. 
In addition, the surface must satisfy conditions on the annulus boundaries of the form

$$
\begin{aligned}
& f\left(r_{i}, \theta\right)=F_{i}(\theta), \quad i=1,2 \\
& \left.\frac{\partial f}{\partial r}\right|_{r=r_{i}}=G_{i}(\theta), \quad i=1,2
\end{aligned}
$$

One such surface that does this is the so-called thin-plate spline, which minimises the functional

$$
J(f)=\int_{\Omega}\left\{f_{x x}^{2}+2 f_{x y}^{2}+f_{y y}^{2}\right\} d \mathbf{x}
$$

subject to the constraints

$$
f\left(\mathbf{x}_{m}\right)=z_{m}, \quad m=1, \ldots, M .
$$

This is equivalent to finding the solution of the inhomogeneous biharmonic equation

$$
\nabla^{4} f+\sum_{m=1}^{M} \lambda_{m} \delta\left(\mathbf{x}-\mathbf{x}_{m}\right)=0
$$

Now the Green's function solution for the biharmonic equation

$$
\nabla^{4} G(\mathbf{x})=\delta\left(\mathbf{x}-\mathbf{x}_{0}\right)
$$

is

$$
H\left(\mathbf{x} ; \mathbf{x}_{\mathbf{0}}\right)=\frac{1}{16 \pi}\left\|\mathbf{x}-\mathbf{x}_{0}\right\|^{2} \log \left\|\mathbf{x}-\mathbf{x}_{0}\right\|^{2}
$$


Given this solution, it is convenient to write the general solution to (3) as

$$
f(\mathbf{x})=g_{0}(\mathbf{x})+\sum_{m=1}^{M} \lambda_{m}\left[g_{m}(\mathbf{x})+H\left(\mathbf{x} ; \mathbf{x}_{m}\right)\right]
$$

and then solve the homogeneous biharmonic equations

$$
\nabla^{4} g_{m}=0, \quad m=0, \ldots, M
$$

with boundary conditions

$$
\begin{array}{rrrrr}
g_{0}\left(r_{i}, \theta\right) & =F_{i}(\theta), & i=1,2 & \\
g_{m}\left(r_{i}, \theta\right) & = & -H_{m, i}(\theta), & i=1,2, & m=1, \ldots, M \\
\left.\frac{\partial g_{0}}{\partial r}\right|_{r=r_{i}} & = & G_{i}(\theta) & i=1,2 & \\
\left.\frac{\partial g_{m}}{\partial r}\right|_{r=r_{i}} & = & -K_{m, i}(\theta) & i=1,2, & m=1, \ldots, M
\end{array}
$$

where

$$
H_{m, i}(\theta)=H\left(r_{i}, \theta ; \mathbf{x}_{m}\right), \quad K_{m, i}(\theta)=\left.\frac{\partial H\left(r, \theta ; \mathbf{x}_{m}\right)}{\partial r}\right|_{r=r_{i}} .
$$

We write the general solution for $g_{0}(r, \theta)$ as

$$
\begin{aligned}
& g_{0}(r, \theta) \\
= & a_{00}+b_{00}\left(\frac{r}{r_{2}}\right)^{2}+c_{00} \log \left(\frac{r_{1}}{r}\right)+d_{00}\left(\frac{r}{r_{2}}\right)^{2} \log \left(\frac{r_{1}}{r}\right) \\
& +\left[a_{10}\left(\frac{r}{r_{2}}\right)^{3}+b_{10}\left(\frac{r}{r_{2}}\right)+c_{10}\left(\frac{r_{1}}{r}\right)+d_{10}\left(\frac{r}{r_{2}}\right) \log \left(\frac{r_{1}}{r}\right)\right] \sin \theta
\end{aligned}
$$




$$
\begin{aligned}
& +\left[e_{10}\left(\frac{r}{r_{2}}\right)^{3}+f_{10}\left(\frac{r}{r_{2}}\right)+h_{10}\left(\frac{r_{1}}{r}\right)+k_{10}\left(\frac{r}{r_{2}}\right) \log \left(\frac{r_{1}}{r}\right)\right] \cos \theta \\
& +\sum_{n=2}^{\infty}\left[a_{n 0}\left(\frac{r}{r_{2}}\right)^{n}+b_{n 0}\left(\frac{r}{r_{2}}\right)^{n+2}+c_{n 0}\left(\frac{r_{1}}{r}\right)^{n}+d_{n 0}\left(\frac{r_{1}}{r}\right)^{n-2}\right] \sin n \theta \\
& +\sum_{n=2}^{\infty}\left[e_{n 0}\left(\frac{r}{r_{2}}\right)^{n}+f_{n 0}\left(\frac{r}{r_{2}}\right)^{n+2}+h_{n 0}\left(\frac{r_{1}}{r}\right)^{n}+k_{n 0}\left(\frac{r_{1}}{r}\right)^{n-2}\right] \cos n \theta
\end{aligned}
$$

and for $g_{m}(r, \theta), m=1, \ldots, M$ as

$$
\begin{aligned}
g_{m}(r, \theta)=a_{0 m} & +b_{0 m}\left(\frac{r}{r_{2}}\right)^{2}+c_{0 m} \log \left(\frac{r_{1}}{r}\right)+d_{0 m}\left(\frac{r}{r_{2}}\right)^{2} \log \left(\frac{r_{1}}{r}\right) \\
+ & {\left[e_{1 m}\left(\frac{r}{r_{2}}\right)^{3}+f_{1 m}\left(\frac{r}{r_{2}}\right)\right.} \\
& \left.+h_{1 m}\left(\frac{r_{1}}{r}\right)+k_{1 m}\left(\frac{r}{r_{2}}\right) \log \left(\frac{r_{1}}{r}\right)\right] \cos \left(\theta-\theta_{m}\right) \\
+ & \sum_{n=2}^{\infty}\left[e_{n m}\left(\frac{r}{r_{2}}\right)^{n}+f_{n m}\left(\frac{r}{r_{2}}\right)^{n+2}\right. \\
& \left.+h_{n m}\left(\frac{r_{1}}{r}\right)^{n}+k_{n m}\left(\frac{r_{1}}{r}\right)^{n-2}\right] \cos n\left(\theta-\theta_{m}\right)
\end{aligned}
$$

The above equations are scaled in such a way as to ensure that the terms involving $r$ remain bounded as $n \rightarrow \infty$, and the coefficients themselves remain bounded. It also turns out that this scaling ensures that the conditioning of the matrix problems to be solved remains reasonable, even for quite large values of $n$. Note that only cosine terms are required for the $g_{m}$ 's, as described below. 
The infinite series is truncated at a finite value, $n=N$, in order to obtain an approximation to the true thin-plate spline solution. For $g_{0}$ we then need to determine $8 N+4$ coefficients, while for the other $g_{m}$ 's we need $4 N+4$ coefficients.

The coefficients in the solution for each of the $g_{m}$ are obtained by evaluating the boundary conditions (10). In order to do this, we need a Fourier decomposition of the fundamental solutions, $H_{m, i}(\theta)$ and their radial derivatives, $G_{m, i}(\theta)$. In terms of $r$ and $\theta$ we write

$$
\left\|\mathbf{x}-\mathbf{x}_{m}\right\|^{2}=\frac{r^{2}+r_{m}^{2}-2 r r_{m} \cos \left(\theta-\theta_{m}\right)}{r_{2}^{2}}
$$

where distances are scaled with $r_{2}$. Hence $H_{m, i}(\theta)$ and $G_{m, i}(\theta)$ are even functions of $\theta-\theta_{m}$, so the Fourier decomposition in $\theta$ can be evaluated as a cosine series in $\theta-\theta_{m}$. The most straightforward way to do this is via a Fast Fourier Transform. The first $N$ coefficients of the Fourier series are required, so the functions are evaluated at the $N$ equispaced points on the $\theta-\theta_{m}$ interval $[0,2 \pi)$, defined by

$$
\theta_{k}-\theta_{m}=\frac{2(k-1) \pi}{N}, \quad k=1, \ldots, N
$$

and the FFT cosine transform directly yields the Fourier coefficients. The accuracy of the results is affected by aliasing, so $N$ must be made sufficiently large that all the "power" of the function is accounted for.

Of course, we also need a Fourier decomposition of the boundary condition functions $F_{i}(\theta)$ and $G_{i}(\theta)$. In general, these will not be even functions of $\theta$, so 
the full FFT is required, necessitating evaluation of these functions at $2 N+1$ equispaced points on the interval $[0,2 \pi)$.

For each $g_{m}$, and at each frequency in the Fourier series, the 4 boundary conditions of equation (10) yield a $4 \times 4$ matrix problem to be solved. The matrices are the same for all of the $g_{m}$ 's, so only $N+14 \times 4$ matrices need to be inverted, and all the coefficients for all the $g_{m}$ 's can be evaluated by multiplication with the appropriate right hand side vectors, obtained from the cosine transforms described above. This is a very simple and efficient procedure.

An interesting point is that the coefficients of the $g_{m}$ 's will be the same for all points that have the same value of $r$. So, if the interpolation points are located on a set of concentric circles, the coefficients only need to be evaluated for one point on each circle. This gives the potential to increase the efficiency even further.

The parameters $\lambda_{m}, m=1, \ldots, M$ are then evaluated by ensuring that the function interpolates all the $M$ points. This can be written as the set of equations

$$
\sum_{m=1}^{M} \lambda_{m}\left[g_{m}\left(\mathbf{x}_{l}\right)+H\left(\mathbf{x}_{l} ; \mathbf{x}_{m}\right)\right]=z_{l}-g_{0}\left(\mathbf{x}_{l}\right), \quad l=1, \ldots, M
$$

which is a matrix equation, which we write as

$$
(\mathbf{G}+\mathbf{H}) \boldsymbol{\lambda}=\mathbf{z}-\mathbf{g}_{0}
$$


The matrix $\mathbf{H}$ is symmetric, but $\mathbf{G}$ is not. In this study, we have not taken any special care about the numerical solution of equation (12), since we are only interested in moderate values of $M$ (of order of 1000, say). Thus, standard LINPACK routines using Gaussian elimination with pivoting provide satisfactory solutions.

The advantage of the formulation used here is that the determination of the $\lambda_{m}$ 's is de-coupled from the coefficients required for the solutions of the homogeneous biharmonic equations. This means that the largest matrix to be inverted is the matrix $\mathbf{G}+\mathbf{H}$ and this is a relatively well conditioned matrix. It is possible to obtain a solution in a way that couples the two sets of problems together, but this results in the solution of a much larger matrix, whose conditioning deteriorates as $N$ increases.

\section{Interpolation on a circle}

A natural development of the method is to remove the inner boundary so that the geometry is now a circle. The existing method is easily modified for interpolation on a circle, by removing the boundary conditions at $r=r_{1}$ and then modifying the general solution for the functions $g_{m}$ so that they and their first and second derivatives remain finite at $r=0$. The solutions then take the form:

$$
g_{0}(r, \theta)=a_{00}+b_{00}\left(\frac{r}{r_{2}}\right)^{2}+\sum_{n=1}^{\infty}\left[a_{n 0}\left(\frac{r}{r_{2}}\right)^{n}+b_{n 0}\left(\frac{r}{r_{2}}\right)^{n+2}\right] \sin n \theta
$$




$$
\begin{aligned}
& +\sum_{n=1}^{\infty}\left[e_{n 0}\left(\frac{r}{r_{2}}\right)^{n}+f_{n 0}\left(\frac{r}{r_{2}}\right)^{n+2}\right] \cos n \theta \\
g_{m}(r, \theta)= & a_{0 m}+b_{0 m}\left(\frac{r}{r_{2}}\right)^{2} \\
& +\sum_{n=1}^{\infty}\left[e_{n m}\left(\frac{r}{r_{2}}\right)^{n}+f_{n m}\left(\frac{r}{r_{2}}\right)^{n+2}\right] \cos n\left(\theta-\theta_{m}\right)
\end{aligned}
$$

so there are only half as many coefficients for the $g_{m}$ 's to evaluate. They are evaluated in exactly the same way as before, but only boundary conditions at $r=r_{2}$ need to be satisfied. This means that only $2 \times 2$ matrices need to be inverted, which can be done explicitly.

\section{Examples}

For the annulus geometry, we use the function

$$
f(r, \theta)=\left(r-r_{1}\right)^{2}\left(r_{2}-r\right)^{2} \theta(2 \pi-\theta)(\pi-\theta)
$$

as a simple test function. Notice that

$$
f\left(r_{1}\right)=f\left(r_{2}\right)=\left.\frac{\partial f}{\partial r}\right|_{r=r_{1}}=\left.\frac{\partial f}{\partial r}\right|_{r=r_{2}}=0
$$

and so

$$
g_{0}(r, \theta)=0 .
$$


We test the interpolation using the above method by randomly locating $M$ points on the domain and doing the interpolation with a fixed value of $N$.

Figure 1 shows results for $r_{1}=0.5, r_{2}=1$ and $M=100$ with $N=$ $8,16,32$ and 64 . The plots show that the function is well represented at internal points for $N$ as small as 16, but that near the boundary the function shows considerable variation until $N$ is quite large (greater than 32). So the choice of an appropriate value of $N$ appears to be controlled by the ability of the method to satisfy the boundary conditions. In order to examine the effect of varying $N$ on the boundary conditions, we plot an estimate of the error in the boundary condition on $f$ at $r=r_{2}$, given by the mean square error quantity

$$
E^{2}=\frac{1}{2 \pi} \int_{0}^{2 \pi} f\left(r_{2}, \theta\right)^{2} d \theta
$$

which can be evaluated numerically, and is plotted in Figure 2, for a range of values of $N$.

This figure shows, as we would expect, a general decline in $E$ as $N$ increases, but there is a lot of variation in $\mathrm{E}$ for low to moderate values of $N$. The reason for this lies in the location of the interpolation points (see Figure 1). There are a few of the 100 interpolation points that are very close to the boundaries. Because of this, a large value of $N$ is required to obtain an accurate Fourier series representation of $H_{m, i}(\theta)$ and $K_{m, i}(\theta)$. If $N$ is chosen to be too small, then aliasing will cause error in the Fourier coefficients, which in turn cause error in the coefficients of the $g_{m}$ 's. The function will still interpolate the interpolation points, but it will not satisfy the boundary 

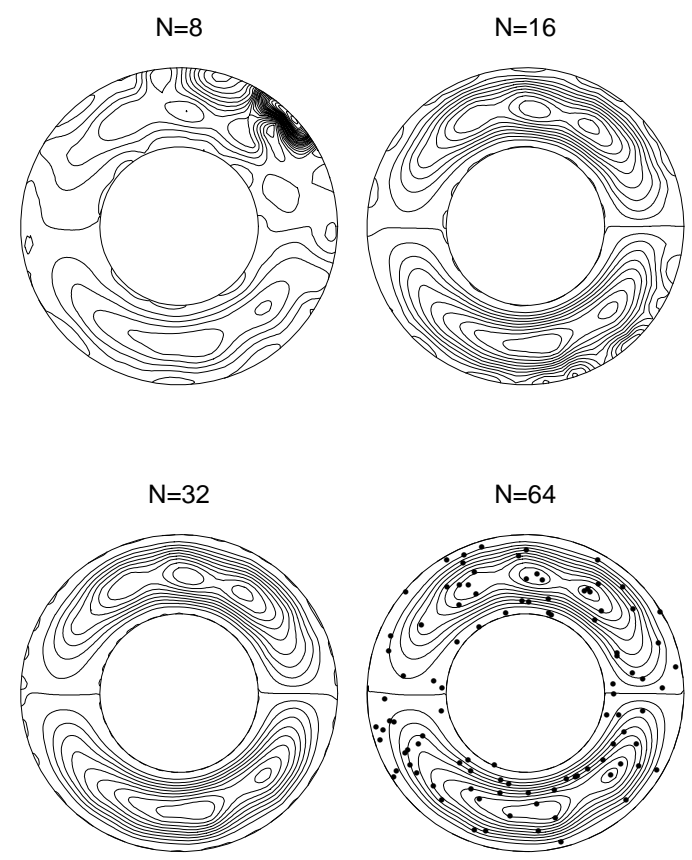

Figure 1: Contour plots of the interpolation function on an annulus, with $r_{1}=0.5, r_{2}=1$ and $M=100$, for various values of $N$. The locations of the 100 interpolation points are shown by the dots on the $N=64$ plot. 


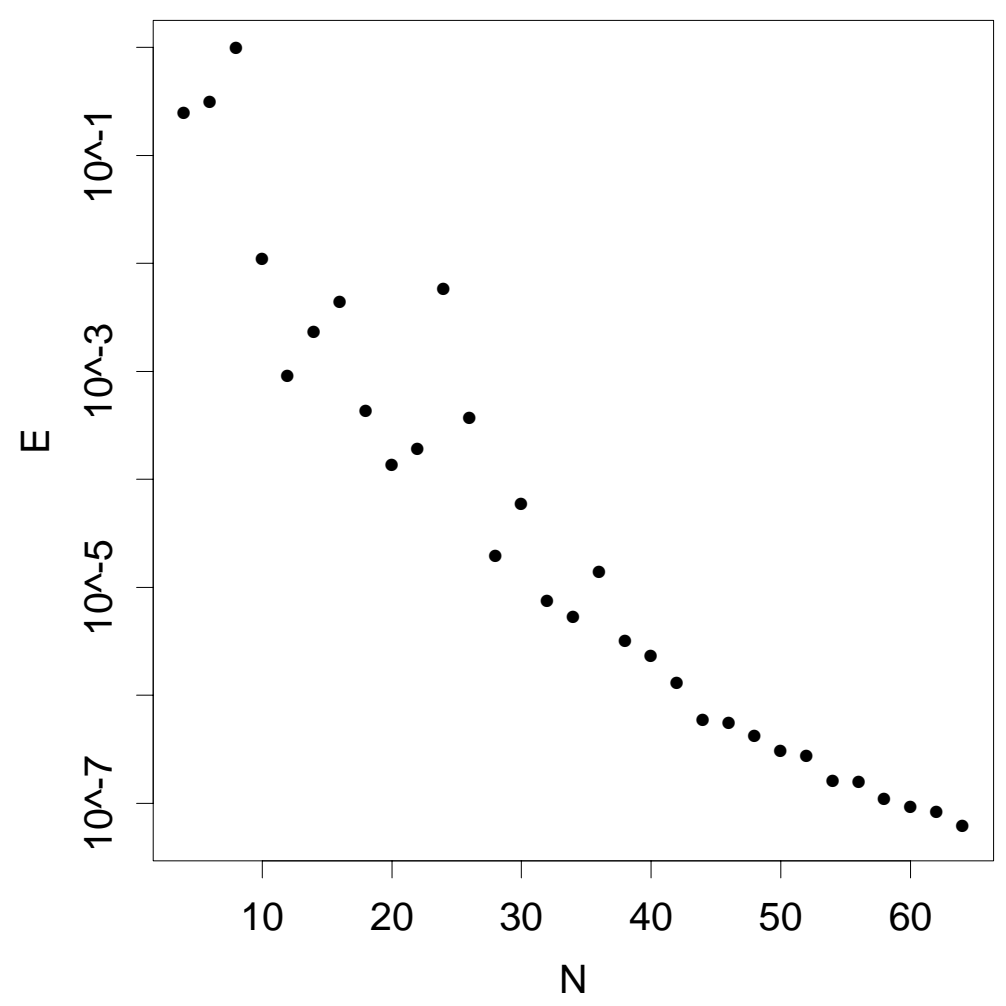

Figure 2: Graph of $E$ versus $N$ for the annulus interpolation function, with $r_{1}=0.5, r_{2}=1$ and $M=100$. 
conditions and it will not be a particularly good representation of the surface elsewhere. Of course, the effect of this type of error is non-local, due to the nature of the series solution approach, so the only solution is to increase $N$.

For the circular geometry, we use the function

$$
f(r, \theta)=r\left(r_{2}-r\right)^{2} \theta(2 \pi-\theta)(\pi-\theta)
$$

as a simple test function. In this case

$$
f\left(r_{2}\right)=\left.\frac{\partial f}{\partial r}\right|_{r=r_{2}}=0
$$

and so, again

$$
g_{0}(r, \theta)=0
$$

Figure 3 shows results for $r_{2}=1$ and $M=100$ with $N=2,4,6$ and 8 . The results here appear to be somewhat better than for the annular example, in that the boundary conditions are satisfied more closely for lower values of $N$. A reasonably good surface is obtained for $N=8$, and even the result for $N=2$ appears quite good. The functional form used here is, in some sense, a less difficult test than the annular example, as it is flatter over a larger range of $r$ near the boundary than was the case for the annulus. Also, the removal of the inner boundary means that there are no long range effects of the aliasing in the Fourier coefficients of points located very close to the inner boundary. This seems to make the outer boundary condition easier 

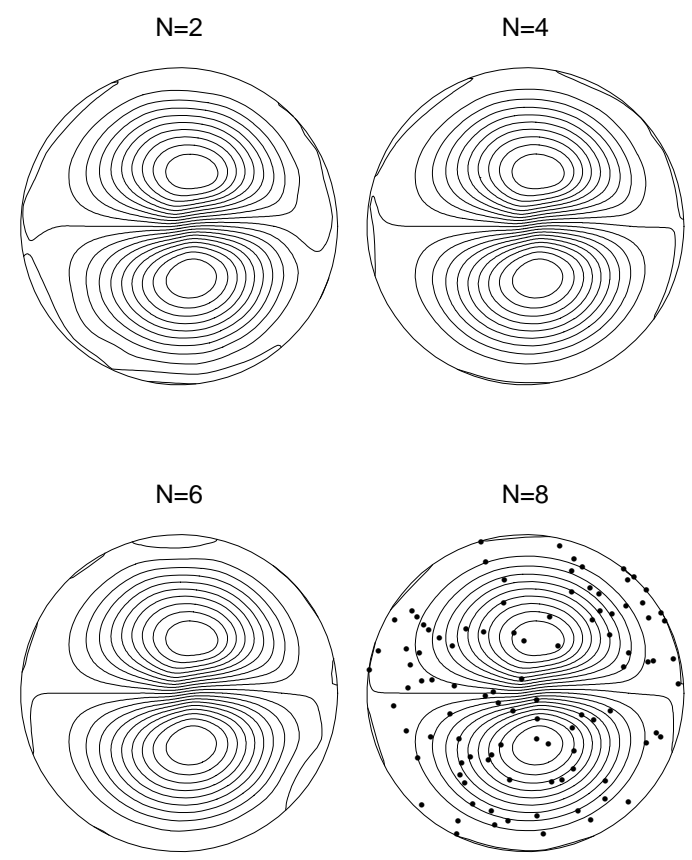

Figure 3: Contour plots of the interpolation function on a circle, with $r_{2}=1$ and $M=100$, for various values of $N$. The locations of the 100 interpolation points are shown by the dots on the $N=8$ plot. 
to satisfy. It is worth noting that Stone [4] found that $N=7$ provided reasonably accurate solutions to his finite domain interpolation problems, and this accords quite well with the results here.

\section{Conclusion}

The method presented here provides a means of using the thin-plate spline approach in situations where the function must conform to specified conditions on the boundary of a circular or annular domain. Moreover, the circular geometry is amenable to a neat formulation of the problem that can be exploited for improving the efficiency of the numerical evaluation of the interpolating surface. There is clearly more that can be done to improve the numerical efficiency of the solution. In particular, no effort has been made to develop efficient means of solving the matrix equation arising from the interpolation conditions. This is not really necessary for the moderate sized interpolation problems addressed here, but may well be necessary for larger problems. Finally, although it has not been addressed here, the method should be able to be adapted for the problem of finding a thin-plate spline "smoothing" surface for noisy data, in the case when boundary conditions are required.

Acknowledgements: The author thanks Drs G. Stone and F. de Hoog, both of CSIRO Mathematical and Information Sciences, for valuable discus- 
sions on this problem.

\section{References}

[1] N. Dyn and D. Levin D. Construction of surface spline interpolants of scattered data over finite domains. R.A.I.R.O. Analyse numérique, 16(3):201-209, 1982. C821

[2] P. J. Green and B. W. Silverman. Nonparametric Regression and Generalized Linear Models: A Roughness Penalty Approach, chapter 7. Chapman \& Hall, 1994. C821

[3] M. Hegland, S. Roberts, and I. Atlas. Finite element thin plate splines for surface fitting. In J. Noye, M. Teubner, and A Gill, editors, Computational Techniques and Applications: CTAC97, pages 209-216, Singapore, 1998. World Scientific. C822

[4] G. Stone. Bivariate Splines. PhD thesis, University of Bath, UK, 1988. C821, C822, C835

[5] P. Wessel and D. Bercovici. D. Intepolation with splines in tension: a Green's function approach. Mathematical Geology, 30(1):77-93, 1998. C822 